\title{
ENTONACIÓN IDIOMÁTICA Y FONÉTICA FORENSE: LA INFORMACIÓN DIALECTAL Y SOCIOLINGÜÍSTICA DE LA ENTONACIÓN EN ESPAÑOL ${ }^{1}$
}

\author{
IDIOMATIC INTONATION AND FORENSIC PHONETICS: SOCIOLINGUISTIC AND \\ DIALECTAL INFORMATION FROM INTONATION IN SPANISH
}

\author{
Yolanda CONGOSTO MARTín \\ Universidad de Sevilla
}

\section{RESUMEN:}

Las investigaciones de carácter geoprosódico realizadas sobre el español y sus variedades ponen en evidencia que la entonación puede reportar importantes beneficios a la fonética forense, ya que esta lleva asociada información de carácter dialectal y sociolingüístico de especial relevancia sobre el individuo - grupo sociocultural al que pertenece, origen geográfico, medio social en el que se inserta-, además de otros rasgos, cualidades o circunstancias como la edad, el sexo, o incluso su temperamento, carácter o estado de ánimo. Para ello se hace necesario no solo saber qué parámetros acústicos suprasegmentales son relevantes para la identificación del hablante, sino también analizar la variación intra e inter locutor, con objeto de poder describir los rasgos que caracterizan al individuo (Battaner et al., 2003). De acuerdo con lo expuesto, el propósito del estudio que aquí presentamos es comprobar el papel que juega la entonación en el reconocimiento de elementos lingüísticos, sociolingüísticos y dialectales. En concreto, se pretende comprobar si los jueces seleccionados son capaces de identificar/discriminar entre: a) modalidades oracionales (aseverativas vs. interrogativas); b) entre variedades dialectales (español mexicano vs. mexicoamericano). Para ello se utilizarán los ficheros de síntesis (archivos de audios solo con información suprasegmental, sin contenido léxico) generados por el programa Amper 2006 (elaborado en el entorno Matlab).

PALABRAS CLAVE: prosodia, entonación, reconocimiento de locutor, variación lingüística, geolingüística, sociolingüística, dialectología, fonética acústica, fonética perceptiva, fonética forense.

\section{ABSTRACT:}

Geographic and prosodic investigations about Spanish and its varieties show how Phonetics can report benefits to Phorensic Phonetics, because of his sociolinguistic character (showing sociocultural group, geographic nature, age, sex...). For this reason, it is required to know both suprasegmental acoustic parameters and intra/inter speaker variation to identify subjects (Battaner et al. 2003). According to this, the aim of this paper is to check the role of intonation

\footnotetext{
${ }^{1}$ Este artículo forma parte de las investigaciones que se están realizando dentro del Proyecto de Investigación PROESLA, Características prosódicas del español de los Estados Unidos: Los Ángeles, California”, financiado por el Ministerio de Economía y Competitividad (Ref.: FFI2011-23292).
} 
to recognize linguistic, sociolinguistic and dialectal elements. Concretely, it is expected to determine if selected evaluators are able to identify between: a) sentence modality (affirmatives vs. interrogative); b) dialectal varieties (Mexican Spanish vs. mexicoamerican). In order to do this, synthesis files will be used (files with just suprasegmental information, without lexical content), generated with Amper2006 software (developed with Matlab Environment).

KEY WORDS: Prosody, intonation, speaker identification, linguistic variation, Sociolinguistics, Acoustic Phonetics, Phorensic Phonetics. 


\section{INTRODUCCIÓN}

Las investigaciones de carácter geoprosódico realizadas sobre el español y sus variedades ponen en evidencia que la entonación puede reportar importantes beneficios a la fonética forense, ya que esta lleva asociada información de carácter dialectal y sociolingüístico de especial relevancia sobre el individuo -grupo sociocultural al que pertenece, origen geográfico, medio social en el que se inserta-, además de otros rasgos, cualidades o circunstancias como la edad, el sexo, o incluso su temperamento, carácter o estado de ánimo. Es por ello que cada día son más los trabajos de investigación que ponen de manifiesto la necesidad de incluir información de carácter suprasegmental en sistemas de reconocimiento de locutor, ya que puede aportar mejoras importantes en su funcionamiento (Escudero et al., 2003). Para ello se hace necesario no solo saber qué parámetros acústicos suprasegmentales son relevantes para la identificación del hablante, sino también analizar la variación intra e inter locutor, con objeto de poder describir los rasgos que caracterizan al individuo (Battaner et al., 2003).

En este sentido, y con la intención de comprobar la eficacia lingüística de la entonación en la discriminación de patrones melódicos, se están realizando en el seno del proyecto AMPER estudios perceptivos que tienen como objetivo validar la metodología empleada y los datos obtenidos, así como comprobar si se puede discriminar entre individuos adscritos a una misma variedad, entre variedades dialectales o entre lenguas (Marrero, 2013; Fernández Planas et al., en prensa). Para ello se han diseñado tests de percepción y programas informáticos que miden las distancias prosódicas (Interlandi y Romano, 2004, Romano et al., 2011).

De acuerdo con lo expuesto, el propósito del estudio perceptivo que aquí presentamos es comprobar el papel que juega la entonación en el reconocimiento de elementos lingüísticos, sociolingüísticos y dialectales. En concreto, se pretende comprobar si los jueces seleccionados son capaces de identificar/discriminar entre dos variedades dialectales de una misma lengua: español mexicano vs. mexicoamericano. Los resultados nos permitirán avanzar en la investigación que se está realizando en el proyecto PROESLA sobre la singularidad y/o continuidad prosódica del español angelino mexicoamericano con respecto al español mexicano, y el posible influjo del inglés sobre la prosodia del español en este ámbito geográfico.

\section{ESTUDIO PERCEPTIVO}

\subsection{Metodología}

\subsubsection{Informantes, puntos de encuesta, jueces y selección de estímulos}

En función de los objetivos, los datos acústicos utilizados para las pruebas de percepción proceden de las bases de datos creadas por los grupos de trabajo AMPERUSA-LA y AMPER-México. En concreto, se han seleccionado las emisiones de dos 
mujeres, una por cada ámbito de estudio; aquellas que responden a las etiquetas AO31 y AX11: la primera es de origen mexicano, residente en Los Ángeles (LA) desde hace 12 años, primera generación de emigrantes y bilingüe de español (mexicano)-inglés; la segunda es natural de México D. F., residente en México (Mx) y monolingüe. Tanto una como otra tienen una edad comprendida entre 25 y 55 años y un nivel de instrucción medio-alto.

Por su parte, los jueces seleccionados han sido en total 16: 9 naturales de México D. F. (M) y 7 procedentes de otros países o ciudades de habla hispana, especialmente de España (NM). Con ello se pretendía saber hasta qué punto el lugar de origen de los jueces y consecuentemente su mayor o menor grado de afinidad con las dos variedades objeto de estudio eran pertinentes para los resultados de las pruebas. Otros factores que también se han tenido en cuenta son la edad y los conocimentos en estudios fónicos, para poder valorar igualmente su posible incidencia y oportunidad en este tipo de estudio. Los jueces mexicanos son alumnos o personas vinculadas al COLMEX (El Colegio de México) y los jueces no mexicanos son alumnos del posgrado en Estudios Fónicos del CSIC/UIMP, en concreto, alumnos del Máster en Fonética y Fonología, Especialidad: Fonética Judicial.

En cuanto al corpus, este ha sido elaborado con los estímulos sintetizados de habla «natural», no manipulada, generados por el programa Amper2006, creado en el entorno Matlab 7.0.4., que es el que se utiliza en el proyecto Amper-español. Se trata de estímulos desprovistos de cualquier tipo de información de carácter léxico-semántico o pragmático, si bien conservan las características prosódicas de las oraciones originales. Estos se obtuvieron a partir del análisis de las 9 oraciones aseverativas y las 9 oraciones interrogativas absolutas sin expansión (del tipo SVO) procedentes del corpus experimental fijo de AMPER, que contienen tres acentos tonales: el patrón acentual del verbo es siempre llano, mientras que el del sujeto y el objeto contemplan todas las posibilidades acentuales del español: aguda, llana y esdrújula ${ }^{2}$. Dado que cada una de ellas se graba (y se analiza) tres veces, el número de estímulos asciende a 27 asev. +27 interr. $=54$ archivos de estímulos sintetizados por informante. A estos se han añadido los archivos extensión.0, resultado de la media de cada tres repeticiones. En definitiva, el número de estímulos seleccionados por informante ha sido de $72(54+18.0=72)$, que por dos informantes hacen un total de 144 estímulos.

\subsubsection{Tests de percepción}

Según la propuesta inicial de Liberman et al. (1957), la identificación modula la discriminación en la percepción del habla humana. Esto significa que si bien en otros modos auditivos (en relación con la percepción de los sonidos del entorno, no del habla) los humanos discriminamos mejor que identificamos, en el «modo del habla» (los autores proponen que nuestra percepción del habla es, de alguna manera, especial), los humanos discriminamos peor que identificamos; al menos, se podría decir que algunos

\footnotetext{
${ }^{2}$ Las grabaciones fueron realizadas con una grabadora digital a $44.1 \mathrm{kHz}, 16$-bit. y analizadas según el protocolo que se sigue en AMPER (Fernández Planas, 2005).
} 
estudios han mostrado que la capacidad de identificación predice el éxito en la capacidad de discriminación.

Así pues, teniendo en cuenta estas premisas, el estudio perceptivo que hemos diseñado consiste en llevar a cabo tres experimentos: un primer experimento de identificación (categorización), seguido de dos experimentos de discriminación. Con ello se pretende que los resultados del primero garanticen el éxito del segundo, y los de ambos, a su vez, el del tercero ${ }^{3}$.

\section{Experimento 1. Test de identificación}

Tiene como objetivo garantizar que los jueces son capaces de diferenciar perceptivamente entre una aseveración y una pregunta (enunciados aseverativos e interrogativos). Para su diseño se han utilizado los 18 estímulos extensión .0 de cada informante, es decir, 36 estímulos. La sesión está compuesta de 36 pruebas y está dividida en cuatro bloques ( 9 pruebas en cada uno), con un descanso entre ellas. Dentro de cada prueba hay un componente de sonido (un estímulo), un componente de texto («te suena como») y tres componentes de respuesta [aseveración (A) / pregunta (B) / no puedo identificarla (C)]. Cada prueba consiste en reproducir (play) un estímulo acústico para que cada oyente/juez lo asocie (pulsar) con uno de los tres objetos (posibilidades) de respuesta, la que considere oportuna. Existe la posibilidad de oír dos veces el estímulo (replay), para los casos de duda. Una vez elegida la respuesta, se puede pasar a la siguiente prueba.

\section{Experimento 2. Test de discriminación simple}

Según la literatura al uso, este es el tipo de experimento de discriminación más sencillo de diseñar. Sin embargo, aunque tiene muchas aplicaciones en el estudio de la psicofísica de la percepción, no tiene tantas en el estudio de la percepción categórica del habla. Además, presenta problemas debidos a la desviación que impone en los jueces.

En cada prueba aparecen dos sonidos emparejados en orden secuencial. Para cada par de sonidos hay una respuesta, con dos alternativas. Se le pregunta al participante: ¿escuchó dos sonidos diferentes o dos instancias del mismo sonido? Aparecen dos teclas: «iguales»-«diferentes». Según los estudios, en este tipo de prueba hay que emparejar los sonidos de manera que haya el mismo número de pares «diferentes» que de pares «iguales». Por ejemplo, para dos sonidos A y B que contrastan de cierta manera hay cuatro pruebas que deben incluirse en el diseño: $\mathrm{AB}, \mathrm{BA}, \mathrm{AA}, \mathrm{BB}$.

El objetivo que queríamos conseguir con este experimento era ver si los jueces eran capaces de asociar dos estímulos (bien interrogativos, bien aseverativos) como «semejantes», es decir, del mismo ámbito dialectal (bien de Mx, bien de LA) o como diferentes (LA/Mx; Mx/LA).

\footnotetext{
${ }^{3}$ Los experimentos han sido diseñados con el programa PRAAT, Version 5.4.
} 


\section{Diseño del experimento 2}

En cada prueba hay un componente de sonido (dos estímulos), un componente de texto («las frases suenan como») y dos componentes de respuesta [semejante/diferente]. Entre un estímulo y otro hay un espacio temporal de $0.5 \mathrm{seg}$. También hay un replay, para los casos de duda, y a cada 12 pruebas, una pausa (4 bloques).

La sesión está formada por 48 pruebas (en 24 se combinan aseverativas con aseverativas; en las otras 24 se combinan interrogativas con interrogativas). En total, 96 estímulos.

Cada prueba consta de dos estímulos, que se han agrupado de la siguiente manera:

- En 3 pruebas, los dos estímulos son del mismo ámbito dialectal (LA-LA): AA

- En otras 3 pruebas, los dos estímulos son también del mismo ámbito dialectal (Mx-Mx): BB

- En 18 pruebas, cada estímulo es de un ámbito dialectal distinto (LA-Mx /MxLA): $\mathrm{AB} / \mathrm{BA}$

Los estímulos se han escogido de la misma selección inicial, de los 144 de que disponíamos. En este caso, se han utilizado las repeticiones 1 y 3 de cada frase (no se ha utilizado la rep. 2). De esta manera:

- En las 6 pruebas en las que los dos estímulos son del mismo ámbito se han utilizado las dos repeticiones ( 1 y 3 ) del mismo informante.

- En las 18 pruebas en las que los dos estímulos son de distinto ámbito se han emparejado:

- bien la rep.1 de un informante con la rep. 1 del otro informante.

- bien la rep. 3 de un informante con la rep. 3 del otro informante.

- bien se ha cruzado la rep.1 con la rep.3, o la rep.3 con la rep.1 (de uno y otro informante).

\section{Experimento 3. Test de discriminación $A X B$}

Este experimento es fundamentalmente diferente de los otros y es probablemente el más usado en los estudios de percepción clásicos. Hay varios diseños diferentes, además de variaciones similares como AXB, ABX, XAB. En él los sonidos se presentan en tríos.

En este caso hemos utilizado el diseño AXB tradicional, según el cual el primer y el tercer sonido siempre son diferentes. El sonido X es siempre «semejante a» uno de los otros dos. La tarea consiste en decir a qué sonido es semejante el sonido X. En resumen, hay tres sonidos y dos respuestas alternativas en cada prueba.

El objetivo que pretendíamos alcanzar con él era seguir analizando el grado de consistencia de los jueces y comprobar, en función de los resultados previos, si el juez seguía asociando los dos estímulos que eran semejantes (mismo informante). Ello nos 
permitiría ver la coherencia en la respuesta del juez en el test 2 y en el test 3, así como el grado de validez de la prueba global.

\section{Diseño del experimento 3}

En cada prueba hay un componente de sonido (tres estímulos), un componente de texto («la segunda frase suena como») y dos componentes de respuesta [Primera/Tercera]. Entre un estímulo y otro hay un espacio temporal de $0.5 \mathrm{seg}$. De nuevo se ha incorporado un replay, para los casos de duda, y a cada 8 pruebas se ha introducido una pausa (9 bloques).

Para su elaboración se han utilizado las 18 parejas de estímulos que se usaron en el experimento 2 y que contenían un estímulo de cada ámbito [(LA-Mx/Mx-LA): $\mathrm{AB} / \mathrm{BA}$ ]. A estos dos estímulos se les ha añadido la rep. 2 que no se había utilizado previamente, para completar la batería de 3 estímulos. De esta manera, hemos podido configurar 36 pruebas de estímulos aseverativos y otras 36 pruebas con estímulos interrogativos:

- Ejemplos Experimento 2. Parejas AB: A01-B01 (aseverativas) / C01-D01 (interrogativas).

- Ejemplos Experimento 3. Trío AXB: A01-A02-B01 / A01-B02-B01 (aseverativas); C01-C02-D01 / C01-D02-D01 (interrogativas).

En total se han utilizado 216 estímulos (72 pruebas con 3 estímulos cada una: 36 aseverativas +36 interrogativas). No se han mezclado las modalidades, sí los informantes.

\subsection{Datos acústicos}

Previo al diseño y realización de los test de percepción las muestras de habla digitalizadas de ambas informantes fueron sometidas a un análisis acústico, para comprobar y evaluar las diferencias y similitudes que se establecían entre ellas. Asimismo, los resultados fueron comparados con los obtenidos sobre otras variedades del español en las que el equipo también ha trabajado (andaluz y extremeño). Es por ello que los resultados del estudio de percepción realizado tienen como finalidad comprobar si, en efecto, las diferencias y similitudes establecidas desde el punto de vista acústico también se manifiestan desde el punto de vista perceptivo.

A continuación se ofrecen algunas muestras de las curvas melódicas de ambas informantes: corresponden a las frases El saxofón/la guitarralel órgano se toca con pánico, en las dos modalidades, aseverativa e interrogativa (figuras 1 y 2 ). 


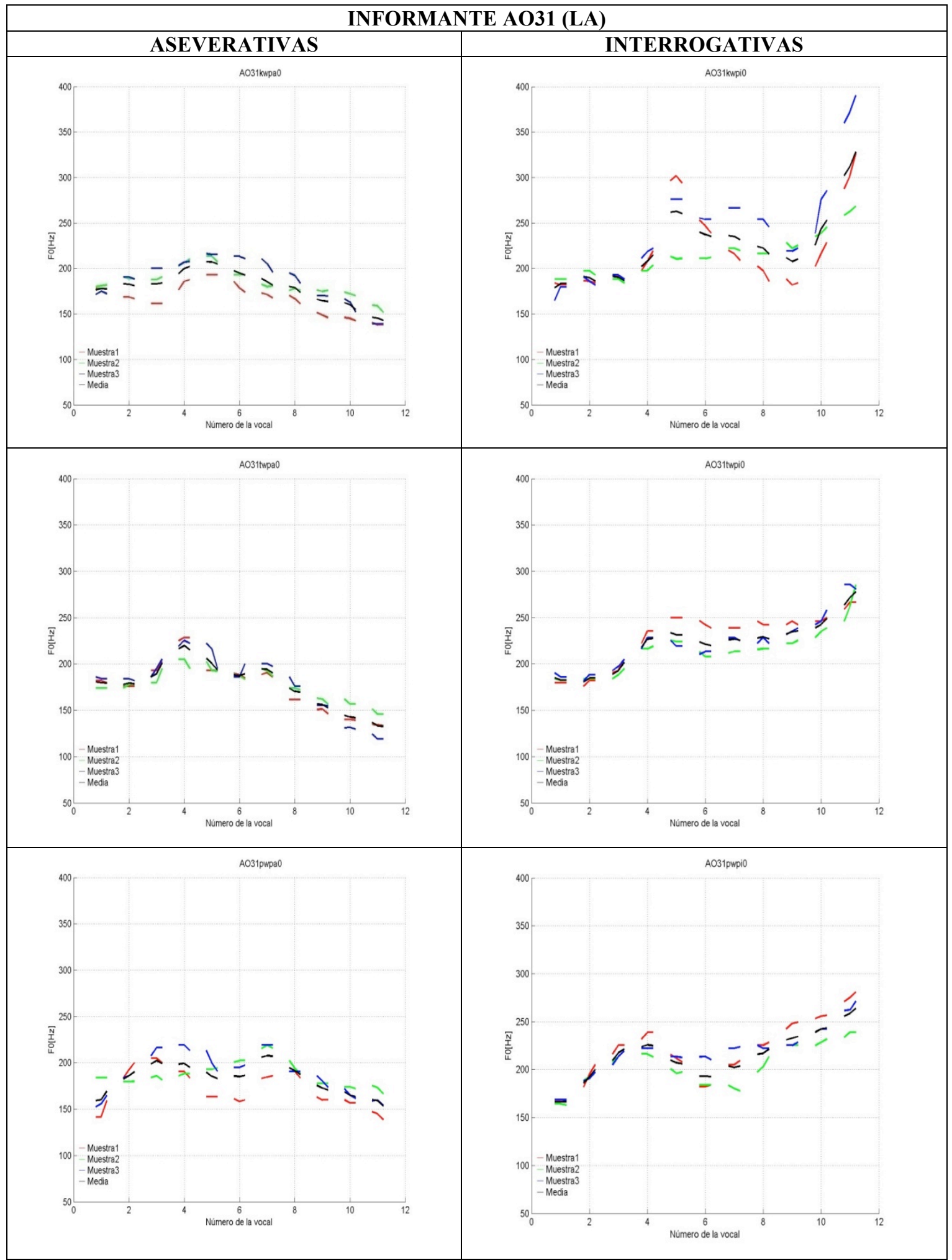

Figura 1. Curvas melódicas de la informante de Los Ángeles 


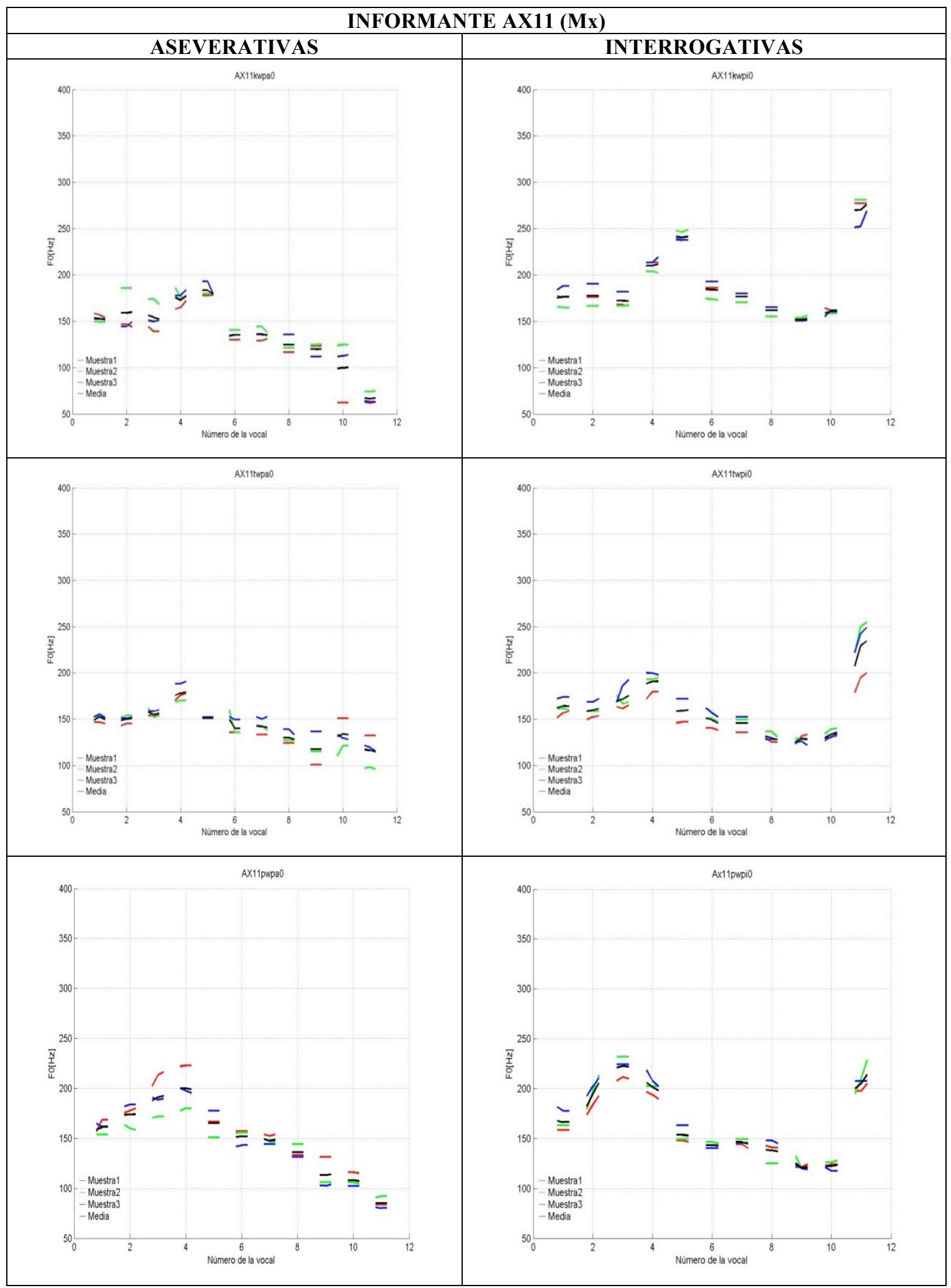

Figura 2. Curvas melódicas de la informante de México 
En el caso de los enunciados aseverativos (figura 3), las curvas melódicas de las dos informantes presentan una configuración tonemática en cadencia $(\mathrm{L} * \mathrm{~L} \%)^{4}$, si bien el descenso del tono es bastante más pronunciado, alcanzando valores más bajos, en la informante mexicana (AX11) que en la mexicoamericana (AO31). En cuanto a la zona pretonemática, todas las curvas presentan un primer pico máximo desplazado $\left(\mathrm{L}+>\mathrm{H}^{*}\right)$ a partir del cual se inicia la declinación, con algunas diferencias significativas, ya que en la informante mexicoamericana se detecta la presencia de un segundo pico ${ }^{5}$, también desplazado, asociado al segundo acento tonal $\left(\mathrm{L}+>! \mathrm{H}^{*}\right)$, inexistente en la curva de la informante mexicana, que hace que el descenso del tono sea más moderado en la primera que en la segunda.

Por lo que respecta a los enunciados interrogativos (figura 3), de nuevo las diferencias melódicas entre ambas informantes empiezan a mostrarse ya en la zona prenuclear de la curva, con distintos recorridos. Mientras que en la informante mexicoamericana podríamos decir que se produce una progresiva y constante subida del tono desde el inicio y hasta el final del enunciado $\left(\mathrm{H}^{*} \mathrm{H} \%\right)$, con un primer pico desplazado $\left(\mathrm{L}+>\mathrm{H}^{*}\right)$, en la informante mexicana la curva melódica se atiene más al patrón tradicional de las interrogativas absolutas en español: subida progresiva del tono desde el inicio y hasta un primer pico, situado en la postónica del primer acento tonal $\left(\mathrm{L}+>\mathrm{H}^{*}\right)$, a continuación una bajada, que culmina en la última sílaba tónica del enunciado, y un final ascedente $\left(\mathrm{L}^{*} \mathrm{HH} \%\right)$, cuya altura en este caso sí que supera la del primer pico. Como se puede apreciar, ambas curvas se inician con movimientos muy similares durante el primer acento tonal, produciéndose después las consabidas diferencias, para volver a encontrarse de nuevo en su tramo final, siempre con valores más altos en AO31 (LA) que en AX11 (Mx).

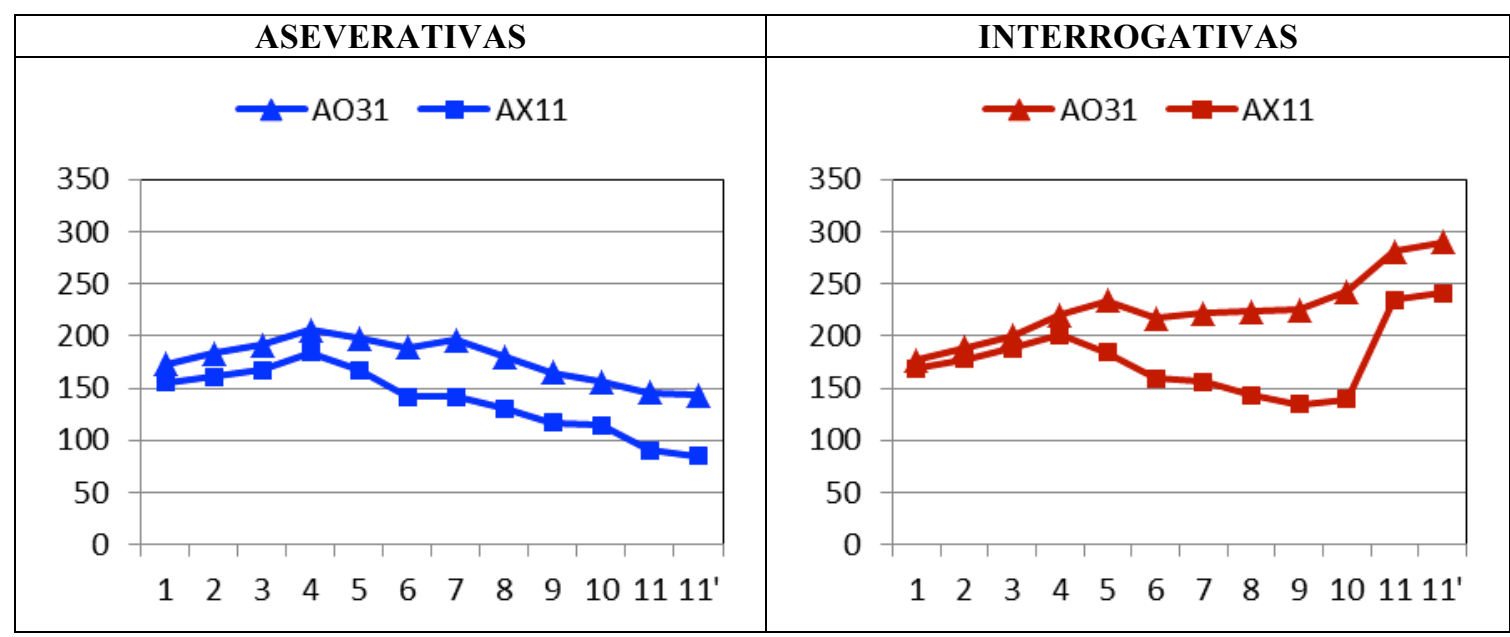

Figura 3. Esquemas de las curvas melódicas de enunciados aseverativos e interrogativos de ambas informantes

\footnotetext{
${ }^{4}$ Para el etiquetaje fonológico se sigue la última propuesta de notación prosódica del español del modelo métrico-autosegmental de análisis entonativo, Sp_TOBI (Estebas y Prieto, 2008).

${ }^{5}$ A excepción del SN sujeto agudo, donde la concatenación de acentos hace que se produzca una cima tonal en la parte central de la curva (ver figura 1).
} 
Finalmente, si superponemos las curvas aseverativas e interrogativas de cada informante y comparamos sus perfiles (figura 4) podremos observar cómo en la informante mexicoamericana las diferencias comienzan ya a manifestarse en la zona central de la curva (segundo acento tonal), mientras que en la informante mexicana no se producen hasta que no se llega a su fin (tonema).

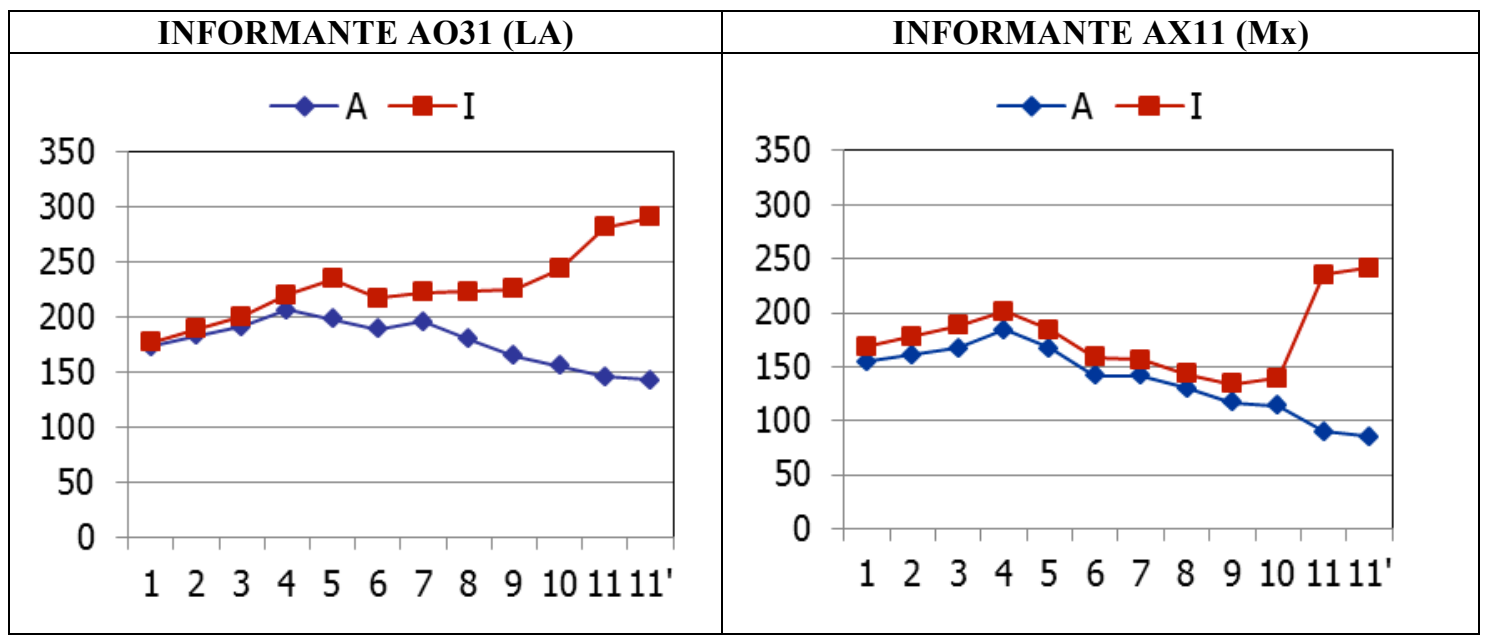

Figura 4. Esquemas superpuestos de las curvas melódicas de enunciados aseverativos e interrogativos de cada informante

\section{RESUltados}

\subsection{Test 1: identificación}

A continuación se aportan tres tablas con los resultados que se han obtenido en el experimento 1 .

Como se puede comprobar, los datos globales (tabla 1.1.) demuestran que los jueces no han tenido problema alguno a la hora de identificar perceptivamente la modalidad oracional que se les ofrecía, ya que el nivel de aciertos ha sido muy alto: $88 \%$ en el caso de los jueces que eran naturales de México y $94 \%$, algo superior, en aquellos que procedían de otras cuidades del ámbito panhispánico.

\begin{tabular}{|l|c|c|c|c|c|}
\hline & \multicolumn{2}{|c|}{ Respuestas correctas } & \multicolumn{2}{c|}{ Respuestas incorrectas } & \multirow{2}{*}{} \\
\cline { 2 - 5 } & N. $^{\mathbf{0}}$ de respuestas & $\mathbf{\%}$ & $\mathbf{N .}^{\mathbf{0}}$ de respuestas & $\boldsymbol{\%}$ & Total respuestas \\
\hline México & 285 & $88 \%$ & 39 & $12 \%$ & 324 \\
\hline No México & 238 & $94 \%$ & 14 & $6 \%$ & 252 \\
\hline
\end{tabular}

Tabla 1.1. Total de respuestas correctas e incorrectas

Si los analizamos con un mayor detenimiento (tablas 1.2. y 1.3.), podemos comprobar que solo dos de los dieciseis jueces seleccionados, en concreto M1 y M8, 
ambos de origen mexicano, han tenido un número de respuestas acertadas por debajo de la media (64\% y $75 \%$, respectivamente), ya que el resto se mueve entre el $86 \%$ de NM1 y el $100 \%$ de M3 y NM4.

Asimismo, no parece que la variable «edad» haya podido influir, ya que si bien M1 ha obtenido un porcentaje bajo (64\%), M7 ha alcanzado el $94 \%$.

En cuanto al grado de instrucción, se partía de la base de que el tener conocimientos de fonética era un factor determinante que incidiría positivamente en los resultados de la prueba; sin embargo, NM1 (+ fon.) ha acertado solo un $86 \%$, frente a M4 y M7 (- fon.) que tienen un $94 \%$. De todas formas, parece obvio que el ser especialista en la materia garantiza el éxito (a excepción de NM1, los nueve estudiantes restantes (+ fon.) superan el $90 \%$ de aciertos).

\begin{tabular}{|c|c|c|c|c|c|c|c|c|c|}
\hline MÉXICO & M1 & M2 & M3 & M4 & M5 & M6 & M7 & M8 & M9 \\
\hline Correctas & 23 & 33 & 36 & 34 & 32 & 34 & 34 & 27 & 32 \\
\hline Incorrectas & 9 & 1 & 0 & 2 & 4 & 1 & 1 & 9 & 3 \\
\hline Dudas & 4 & 2 & 0 & 0 & 0 & 1 & 1 & 0 & 1 \\
\hline Correctas & $64 \%$ & $92 \%$ & $100 \%$ & $94 \%$ & $89 \%$ & $94 \%$ & $94 \%$ & $75 \%$ & $89 \%$ \\
\hline Incorrectas & $25 \%$ & $3 \%$ & $0 \%$ & $6 \%$ & $11 \%$ & $3 \%$ & $3 \%$ & $25 \%$ & $8 \%$ \\
\hline Dudas & $11 \%$ & $6 \%$ & $0 \%$ & $0 \%$ & $0 \%$ & $3 \%$ & $3 \%$ & $0 \%$ & $3 \%$ \\
\hline \multirow{3}{*}{ Observaciones } & $\begin{array}{l}\text { México } \\
\text { D.F. }\end{array}$ & $\begin{array}{c}\text { México } \\
\text { D.F. }\end{array}$ & $\begin{array}{c}\text { México } \\
\text { D.F. }\end{array}$ & $\begin{array}{c}\text { México } \\
\text { D.F. }\end{array}$ & $\begin{array}{c}\text { México } \\
\text { D.F. }\end{array}$ & $\begin{array}{c}\text { México } \\
\text { D.F. }\end{array}$ & $\begin{array}{l}\text { México } \\
\text { D.F. }\end{array}$ & $\begin{array}{c}\text { México } \\
\text { D.F. }\end{array}$ & $\begin{array}{l}\text { México } \\
\text { D.F. }\end{array}$ \\
\hline & mayor & joven & joven & joven & joven & joven & mayor & joven & joven \\
\hline & - & fonetista & fonetista & - & - & fonetista & - & - & - \\
\hline
\end{tabular}

Tabla 1.2. Respuestas de los jueces de México

\begin{tabular}{|c|c|c|c|c|c|c|c|}
\hline NO MÉXICO & NM1 & NM2 & NM3 & NM4 & NM5 & NM6 & NM7 \\
\hline Correctas & 31 & 35 & 35 & 36 & 35 & 33 & 33 \\
\hline Incorrectas & 3 & 1 & 0 & 0 & 1 & 2 & 1 \\
\hline Dudas & 2 & 0 & 1 & 0 & 0 & 1 & 2 \\
\hline Correctas & $86 \%$ & $97 \%$ & $97 \%$ & $100 \%$ & $97 \%$ & $92 \%$ & $92 \%$ \\
\hline Incorrectas & $8 \%$ & $3 \%$ & $0 \%$ & $0 \%$ & $3 \%$ & $6 \%$ & $3 \%$ \\
\hline Dudas & $6 \%$ & $0 \%$ & $3 \%$ & $0 \%$ & $0 \%$ & $3 \%$ & $6 \%$ \\
\hline & $\begin{array}{l}\text { Ibiza } \\
\text { joven }\end{array}$ & $\begin{array}{c}\text { Madrid } \\
\text { joven }\end{array}$ & $\begin{array}{c}\text { Almeria } \\
\text { joven }\end{array}$ & $\begin{array}{c}\text { Madrid } \\
\text { joven }\end{array}$ & $\begin{array}{c}\text { Canarias } \\
\text { joven }\end{array}$ & $\begin{array}{l}\text { Perú } \\
\text { joven }\end{array}$ & $\begin{array}{c}\text { Barcelona } \\
\text { joven }\end{array}$ \\
\hline Observaciones & fonetista & fonetista & fonetista & fonetista & fonetista & fonetista & fonetista \\
\hline
\end{tabular}

Tabla 1.3. Respuestas de los jueces que no son de México

\subsection{Test 2: discriminación simple}

De nuevo las seis tablas que se incluyen en este epígrafe ofrecen los resultados globales y parciales obtenidos en el experimento 2 . 
En este caso (ver tabla 2.1), en contraste con lo ocurrido en el test 1, los índices de aciertos son ligeramente más altos en los jueces de México D. F. (71\%) que en los propios de otras ciudades (69\%). Probablemente ello se deba a que las diferencias entonativas entre ambas variedades (mexicana $v s$. mexicoamericana) no son altamente significativas $\mathrm{y}$, por tanto, son inapreciables por un oído extranjero (ajeno a dicha comunidad de habla); no así para el juez mexicano quien con más facilidad advierte estas pequeñas y sutiles diferencias melódicas. De todas formas, en líneas generales, se podría llegar a la conclusión de que el nivel de errores ha sido quizás más alto de lo esperado.

\begin{tabular}{|l|c|c|c|c|c|}
\hline \multirow{2}{*}{} & \multicolumn{2}{|c|}{ Respuestas correctas } & \multicolumn{2}{c|}{ Respuestas incorrectas } & \multirow{2}{*}{ Total respuestas } \\
\cline { 2 - 5 } & $\mathbf{N .}^{\mathbf{0}}$ de respuestas & $\mathbf{\%}$ & $\mathbf{N .}^{\mathbf{0}}$ de respuestas & $\boldsymbol{\%}$ & \\
\hline México & 308 & $71 \%$ & 124 & $29 \%$ & 432 \\
\hline No México & 195 & $69 \%$ & 93 & $31 \%$ & 336 \\
\hline
\end{tabular}

Tabla 2.1. Total de respuestas correctas e incorrectas

Si pasamos al análisis detallado de los datos, podremos comenzar diciendo que entre los jueces mexicanos (tabla 2.2) se aprecian resultados muy variados, que oscilan entre el $8 \%$ de error de M9, curiosamente (- fon.), y el $46 \%$ de error de M2 (+ fon.); es más, incluso se podría establecer una gradación de errores: $8 \%$ - $13 \%$ - $25 \%-31 \%$ $33 \%-38 \%-46 \%$.

\begin{tabular}{|c|c|c|c|c|c|c|c|c|c|}
\hline MÉXICO & M1 & M2 & M3 & M4 & M5 & M6 & M7 & M8 & M9 \\
\hline Correctas & 36 & 26 & 32 & 33 & 32 & 42 & 32 & 30 & 44 \\
\hline Incorrectas & 12 & 22 & 16 & 15 & 16 & 6 & 16 & 18 & 4 \\
\hline Correctas & $75 \%$ & $54 \%$ & $67 \%$ & $69 \%$ & $67 \%$ & $88 \%$ & $67 \%$ & $63 \%$ & $92 \%$ \\
\hline Incorrectas & $25 \%$ & $46 \%$ & $33 \%$ & $31 \%$ & $33 \%$ & $13 \%$ & $33 \%$ & $38 \%$ & $8 \%$ \\
\hline \multirow{3}{*}{ Observaciones } & $\begin{array}{c}\text { México } \\
D F\end{array}$ & $\begin{array}{c}\text { México } \\
D F\end{array}$ & $\begin{array}{c}\text { México } \\
D F\end{array}$ & $\begin{array}{c}\text { México } \\
D F\end{array}$ & $\begin{array}{c}\text { México } \\
D F\end{array}$ & $\begin{array}{c}\text { México } \\
D F\end{array}$ & $\begin{array}{c}\text { México } \\
D F\end{array}$ & $\begin{array}{c}\text { México } \\
D F\end{array}$ & $\begin{array}{c}\text { México } \\
D F\end{array}$ \\
\hline & mayor & joven & joven & joven & joven & joven & mayor & joven & joven \\
\hline & - & fonetista & fonetista & - & - & fonetista & - & - & - \\
\hline
\end{tabular}

Tabla 2.2. Respuestas de los jueces de México

Por su parte, los jueces de las otras ciudades (tabla 2.3.) presentan de algún modo resultados más homogéneos ya que oscilan entre un $21 \%$ (NM4) y un $46 \%$ (NM1); la mitad de variación si los comparamos con los obtenidos de los jueces mexicanos. También llama la atención el que los valores más altos de aciertos procedan de jueces madrileños (NM2 y NM4). 


\begin{tabular}{|c|c|c|c|c|c|c|c|}
\hline NO MÉXICO & NM1 & NM2 & NM3 & NM4 & NM5 & NM6 & NM7 \\
\hline Correctas & 26 & 37 & 21 & 38 & 22 & 31 & 33 \\
\hline Incorrectas & 22 & 11 & 27 & 10 & 26 & 17 & 15 \\
\hline Correctas & $54 \%$ & $77 \%$ & $62,5 \%$ & $79 \%$ & $62,5 \%$ & $65 \%$ & $69 \%$ \\
\hline \multirow[t]{3}{*}{ Incorrectas } & $46 \%$ & $23 \%$ & $37,5 \%$ & $21 \%$ & $37,5 \%$ & $35 \%$ & $31 \%$ \\
\hline & Ibiza & Madrid & Almería & Madrid & Canarias & Perú & Barcelona \\
\hline & $\begin{array}{c}\text { joven } \\
\text { fonetista }\end{array}$ & $\begin{array}{c}\text { joven } \\
\text { fonetista }\end{array}$ & $\begin{array}{c}\text { joven } \\
\text { fonetista }\end{array}$ & $\begin{array}{c}\text { joven } \\
\text { fonetista }\end{array}$ & $\begin{array}{c}\text { joven } \\
\text { fonetista }\end{array}$ & $\begin{array}{c}\text { joven } \\
\text { fonetista }\end{array}$ & $\begin{array}{c}\text { joven } \\
\text { fonetista }\end{array}$ \\
\hline
\end{tabular}

Tabla 2.3. Respuestas de los jueces de No México

A continuación, la tabla 2.4. ofrece una comparación global por modalidad.

En lo que respecta a las aseverativas, podemos decir que en líneas generales estas han planteado más dudas que las interrogativas. Ello puede deberse a que, como ya quedó demostrado en el estudio acústico, las curvas de los enunciados aseverativos de los dos ámbitos (LA y Mx) no presentan tantas diferencias como las interrogativas; o al menos estas son menos perceptibles al oído, por lo que los jueces han dudado más a la hora de discriminar si se trataba de un mismo informante (y ámbito) o de dos informantes (y ámbitos) distintos.

Las respuestas correctas (tanto cuando los estímulos son semejantes como cuando son diferentes) son muy parejas: en el caso de los jueces de México, $74 \%$ (semejantes) y $72 \%$ (diferentes); en el caso de los jueces de otras ciudades: $69 \%$ (semejantes) y $60 \%$ (diferentes). En efecto, el nivel de aciertos es más alto cuando los jueces son mexicanos, ya que aprecian mejor los diferentes matices entre las aseverativas de uno y otro ámbito.

En cuanto a las interrogativas, es evidente que sus melodías están más definidas: las parejas de estímulos que eran semejantes (del mismo informante) han sido claramente identificadas: $91 \%$ y $93 \%$ respectivamente. Sin embargo, resulta llamativo ver que cuando las parejas de estímulos eran diferentes el porcentaje de aciertos ha sido menor: $62 \%$ (jueces mexicanos) y $70 \%$ (jueces no mexicanos), sobre todo si tenemos en cuenta que las curvas de las dos informantes seleccionadas mostraban notorias diferencias. Quizás el hecho de que estas se produzcan en la zona central de la curva y no en su tramo inicial ni final, donde sí coinciden, sea el motivo que lo justifique. 


\begin{tabular}{|c|c|c|c|c|c|}
\hline \multirow{2}{*}{\multicolumn{2}{|c|}{ ASEVERATIVAS }} & \multicolumn{2}{|c|}{$\begin{array}{l}\text { Mismo informante } \\
\text { (son semejantes) }\end{array}$} & \multicolumn{2}{|c|}{$\begin{array}{c}\begin{array}{c}\text { Distintos informantes } \\
\text { (son diferentes) }\end{array} \\
\end{array}$} \\
\hline & & N. ${ }^{\circ}$ de respuestas & $\%$ & N. ${ }^{\circ}$ de respuestas & $\%$ \\
\hline \multirow{2}{*}{ MÉXICO } & Correctas & 40 & $74 \%$ & 117 & $72 \%$ \\
\hline & Incorrectas & 14 & $26 \%$ & 45 & $28 \%$ \\
\hline \multirow{2}{*}{$\begin{array}{c}\text { NO } \\
\text { MÉXICO }\end{array}$} & Correctas & 29 & $69 \%$ & 75 & $60 \%$ \\
\hline & Incorrectas & 13 & $31 \%$ & 51 & $40 \%$ \\
\hline \multirow{2}{*}{\multicolumn{2}{|c|}{ INTERROGATIVAS }} & \multicolumn{2}{|c|}{$\begin{array}{c}\text { Mismo informante } \\
\text { (son semejantes) }\end{array}$} & \multicolumn{2}{|c|}{$\begin{array}{c}\begin{array}{c}\text { Distintos informantes } \\
\text { (son diferentes) }\end{array} \\
\end{array}$} \\
\hline & & N. ${ }^{\circ}$ de respuestas & $\%$ & N. ${ }^{\circ}$ de respuestas & $\%$ \\
\hline \multirow{2}{*}{ MÉXICO } & Correctas & 49 & $91 \%$ & 101 & $62 \%$ \\
\hline & Incorrectas & 5 & $9 \%$ & 61 & $38 \%$ \\
\hline \multirow{2}{*}{$\begin{array}{c}\text { NO } \\
\text { MÉXICO }\end{array}$} & Correctas & 40 & $93 \%$ & 64 & $70 \%$ \\
\hline & Incorrectas & 2 & $7 \%$ & 62 & $30 \%$ \\
\hline
\end{tabular}

Tabla 2.4. Comparación global de las respuestas de los jueces de México y No México por modalidad

Las dos tablas siguientes comparan los datos por modalidad y por ámbito dialectal (mismo informante o distintos informantes) en relación con los jueces: la 2.5 referente a los jueces de origen mexicano; la 2.6. relativa a los jueces de otros ámbitos.

En este sentido, y en función de los resultados, solo cabe añadir a lo ya dicho que los jueces naturales de Andalucía y de Canarias tuvieron problemas con las interrogativas: o bien estaban cansados y no prestaron suficiente atención, o bien no apreciaron las diferencias, o si las apreciaron no supieron identificarlas, por lo que han sido excluidos de los cálculos de este experimento.

\begin{tabular}{|c|c|c|c|c|c|c|c|c|c|c|}
\hline \multicolumn{2}{|c|}{ MÉXICO } & M1 & M2 & M3 & M4 & M5 & M6 & M7 & M8 & M9 \\
\hline \multirow{2}{*}{$\begin{array}{l}\text { Aseverativas } \\
\text { Mismo inf. }\end{array}$} & Correc. & $50 \%$ & $83 \%$ & $67 \%$ & $100 \%$ & $83 \%$ & $67 \%$ & $83 \%$ & $67 \%$ & $67 \%$ \\
\hline & Incorr. & $50 \%$ & $17 \%$ & $33 \%$ & $0 \%$ & $17 \%$ & $33 \%$ & $17 \%$ & $33 \%$ & $33 \%$ \\
\hline \multirow{2}{*}{$\begin{array}{l}\text { Aseverativas } \\
\text { Distintos inf. }\end{array}$} & Correc. & $100 \%$ & $39 \%$ & $67 \%$ & $56 \%$ & $56 \%$ & $89 \%$ & $78 \%$ & $67 \%$ & $100 \%$ \\
\hline & Incorr. & $0 \%$ & $61 \%$ & $33 \%$ & $44 \%$ & $44 \%$ & $11 \%$ & $22 \%$ & $33 \%$ & $0 \%$ \\
\hline \multirow{2}{*}{$\begin{array}{l}\text { Interrogativas } \\
\text { Mismo inf. }\end{array}$} & Correc. & $67 \%$ & $100 \%$ & $100 \%$ & $83 \%$ & $100 \%$ & $83 \%$ & $100 \%$ & $83 \%$ & $100 \%$ \\
\hline & Incorr. & $33 \%$ & $0 \%$ & $0 \%$ & $17 \%$ & $0 \%$ & $17 \%$ & $0 \%$ & $17 \%$ & $0 \%$ \\
\hline \multirow{2}{*}{$\begin{array}{c}\text { Interrogativas } \\
\text { Distintos inf. }\end{array}$} & Correc & $61 \%$ & $44 \%$ & $56 \%$ & $67 \%$ & $61 \%$ & $94 \%$ & $39 \%$ & $50 \%$ & $89 \%$ \\
\hline & Incorr. & $39 \%$ & $56 \%$ & $44 \%$ & $33 \%$ & $39 \%$ & $6 \%$ & $61 \%$ & $50 \%$ & $11 \%$ \\
\hline \multicolumn{2}{|c|}{ Observaciones } & $\begin{array}{l}\text { México } \\
\text { D.F. } \\
\text { mayor } \\
-\end{array}$ & $\begin{array}{l}\text { México } \\
\text { D.F. } \\
\text { joven } \\
\text { Fon. }\end{array}$ & $\begin{array}{l}\text { México } \\
\text { D.F. } \\
\text { joven } \\
\text { Fon. }\end{array}$ & $\begin{array}{c}\text { México } \\
\text { D.F. } \\
\text { joven } \\
-\end{array}$ & $\begin{array}{c}\text { México } \\
D . F . \\
\text { joven } \\
-\end{array}$ & $\begin{array}{c}\text { México } \\
D . F . \\
\text { joven } \\
\text { Fon. }\end{array}$ & $\begin{array}{l}\text { México } \\
\text { D.F. } \\
\text { mayor } \\
-\end{array}$ & $\begin{array}{l}\text { México } \\
\text { D.F. } \\
\text { joven } \\
-\end{array}$ & $\begin{array}{l}\text { México } \\
\text { D.F. } \\
\text { joven } \\
-\end{array}$ \\
\hline
\end{tabular}

Tabla 2.5. Respuestas de los jueces de México: comparación por modalidad y por ámbito dialectal (mismo informante/distintos informantes) 


\begin{tabular}{|c|l|c|c|c|c|c|c|c|}
\hline \multicolumn{2}{|c|}{ NO MÉXICO } & NM1 & NM2 & NM3 & NM4 & NM5 & NM6 & NM7 \\
\hline \multirow{2}{*}{$\begin{array}{c}\text { Aseverativas } \\
\text { Mismo inf. }\end{array}$} & Correctas & $67 \%$ & $17 \%$ & $83 \%$ & $67 \%$ & $67 \%$ & $100 \%$ & $83 \%$ \\
\cline { 2 - 10 } & Incorrectas & $33 \%$ & $83 \%$ & $17 \%$ & $33 \%$ & $33 \%$ & $0 \%$ & $17 \%$ \\
\hline $\begin{array}{c}\text { Aseverativas } \\
\text { Distintos inf. }\end{array}$ & Correctas & $44 \%$ & $89 \%$ & $56 \%$ & $72 \%$ & $61 \%$ & $44 \%$ & $50 \%$ \\
\hline \hline \multirow{2}{*}{$\begin{array}{c}\text { Interrogativas } \\
\text { Mismo inf. }\end{array}$} & Incorrectas & $56 \%$ & $11 \%$ & $44 \%$ & $28 \%$ & $39 \%$ & $56 \%$ & $50 \%$ \\
\cline { 2 - 11 } & Incorrectas & $17 \%$ & $0 \%$ & $0 \%$ & $0 \%$ & $0 \%$ & $17 \%$ & $0 \%$ \\
\hline $\begin{array}{c}\text { Interrogativas } \\
\text { Distintos inf. }\end{array}$ & Correctas & $50 \%$ & $78 \%$ & $0 \%$ & $83 \%$ & $0 \%$ & $67 \%$ & $72 \%$ \\
\cline { 2 - 11 } & Incorrectas & $50 \%$ & $22 \%$ & $0 \%$ & $17 \%$ & $0 \%$ & $33 \%$ & $28 \%$ \\
\hline
\end{tabular}

Tabla 2.6. Respuestas de los jueces de No México: comparación por modalidad y ámbito dialectal (mismo informante/distintos informantes)

\subsection{Test 3: discriminación $A X B$}

Como se aprecia en la tabla 3.1., el nivel global de aciertos en esta prueba ha alcanzado el 87,5\%, con resultados parecidos en los dos grupos de jueces.

\begin{tabular}{|l|c|c|c|c|c|}
\hline & \multicolumn{2}{|c|}{ Respuestas correctas } & \multicolumn{2}{c|}{ Respuestas incorrectas } & \multirow{2}{*}{$\begin{array}{c}\text { Total de } \\
\text { respuestas }\end{array}$} \\
\cline { 2 - 5 } & N. $^{\mathbf{0}}$ de respuestas & $\mathbf{\%}$ & N. $^{\mathbf{0}}$ de respuestas & \% & 648 \\
\hline MÉXICO & 565 & $87 \%$ & 83 & $13 \%$ & 504 \\
\hline NO MÉXICO & 444 & $88 \%$ & 60 & $12 \%$ & 5 \\
\hline
\end{tabular}

Tabla 3.1. Total respuestas correctas e incorrectas

En el caso de las aseverativas (ver tabla 3.2.), se han producido más aciertos al asociar las curvas de la informante mexicoamericana $(\mathrm{X}=\mathrm{A}): 93 \%$ y $96 \%$, que en el caso de asociar las curvas de la informante mexicana $(\mathrm{X}=\mathrm{B}): 87 \%$ y $80 \%$.

Curiosamente, los jueces de México han acertado más cuando se trataba de la informante A (LA): $93 \%$, que cuando se trataba de B (Mx): $87 \%$.

Por su parte, los jueces que no son de México también han tenido más aciertos al asociar los estímulos de A (LA): $96 \%$, pero menos aciertos que sus colegas mexicanos al asociar los dos estímulos de $\mathrm{B}(\mathrm{Mx}): 80 \%$, probablemente por no pertenecer a la misma comunidad de habla.

En cuanto a las interrogativas, el mayor número de aciertos se ha producido al asociar los estímulos de B (Mx): 89 \% y $91 \%$, frente a los de A (LA): 80 \% y $85 \%$, ligeramente más bajos. En este caso, los jueces que no son de México son los que han presentado un porcentaje ligeramente más alto. 


\begin{tabular}{|l|l|c|c|c|c|}
\hline \multicolumn{2}{|c|}{} & \multicolumn{2}{c|}{ ASEVERATIVAS } & \multicolumn{2}{c|}{ INTERROGATIVAS } \\
\cline { 3 - 6 } \multicolumn{2}{|c|}{} & $\mathbf{A x B}, \mathbf{x}=\mathbf{A}$ & $\mathbf{A x B}, \mathbf{x}=\mathbf{B}$ & $\mathbf{A x B}, \mathbf{x}=\mathbf{A}$ & $\mathbf{A x B}, \mathbf{x}=\mathbf{B}$ \\
\hline \multirow{2}{*}{ MÉXICO } & Correctas & $93 \%$ & $87 \%$ & $80 \%$ & $89 \%$ \\
\cline { 2 - 6 } & Incorrectas & $7 \%$ & $13 \%$ & $20 \%$ & $11 \%$ \\
\hline \multirow{2}{*}{ NO MÉXICO } & Correctas & $96 \%$ & $80 \%$ & $85 \%$ & $91 \%$ \\
\cline { 2 - 6 } & Incorrectas & $4 \%$ & $20 \%$ & $15 \%$ & $9 \%$ \\
\hline
\end{tabular}

Tabla 3.2. Comparación global de las respuestas de los jueces de México y de No México por modalidad y por el origen del informante (A - Los Ángeles / B - México)

Por último, las tablas 3.3. y 3.4. comparan las respuestas de los jueces por modalidad y por origen del informante.

En la primera de ellas (jueces de México), solo habría que resaltar sobre lo ya dicho, el que los dos jueces de mayor edad (- fon.) son los que han tenido un mayor índice de errores.

\begin{tabular}{|c|c|c|c|c|c|c|c|c|c|c|c|}
\hline \multicolumn{3}{|c|}{ MÉXICO } & M1 & M2 & M3 & M4 & M5 & M6 & M7 & M8 & M9 \\
\hline \multirow{4}{*}{ 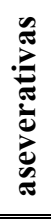 } & \multirow{2}{*}{$\mathbf{A x B}, \mathbf{x}=\mathbf{A}$} & Correctas & $89 \%$ & $100 \%$ & $100 \%$ & $89 \%$ & $89 \%$ & $89 \%$ & $94 \%$ & $94 \%$ & $94 \%$ \\
\hline & & Incorrectas & $11 \%$ & $0 \%$ & $0 \%$ & $11 \%$ & $11 \%$ & $11 \%$ & $6 \%$ & $6 \%$ & $6 \%$ \\
\hline & \multirow{2}{*}{$A x B, x=B$} & Correctas & $83 \%$ & $94 \%$ & $83 \%$ & $94 \%$ & $89 \%$ & $94 \%$ & $67 \%$ & $83 \%$ & $94 \%$ \\
\hline & & Incorrectas & $17 \%$ & $6 \%$ & $17 \%$ & $6 \%$ & $11 \%$ & $6 \%$ & $33 \%$ & $17 \%$ & $6 \%$ \\
\hline \multirow{4}{*}{ 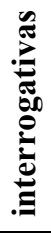 } & \multirow{2}{*}{$\mathbf{A x B}, \mathbf{x}=\mathbf{A}$} & Correctas & $78 \%$ & $83 \%$ & $78 \%$ & $89 \%$ & $78 \%$ & $78 \%$ & $72 \%$ & $78 \%$ & $83 \%$ \\
\hline & & Incorrectas & $22 \%$ & $17 \%$ & $22 \%$ & $11 \%$ & $22 \%$ & $22 \%$ & $28 \%$ & $22 \%$ & $17 \%$ \\
\hline & \multirow{2}{*}{$\mathbf{A x B}, \mathbf{x}=\mathbf{B}$} & Correctas & $61 \%$ & $94 \%$ & $89 \%$ & $100 \%$ & $100 \%$ & $94 \%$ & $78 \%$ & $94 \%$ & $89 \%$ \\
\hline & & Incorrectas & $39 \%$ & $6 \%$ & $11 \%$ & $0 \%$ & $0 \%$ & $6 \%$ & $22 \%$ & $6 \%$ & $11 \%$ \\
\hline & \multicolumn{2}{|c|}{ Observaciones } & $\begin{array}{c}\text { mayor } \\
-\end{array}$ & $\begin{array}{c}\text { joven } \\
\text { fonetista }\end{array}$ & $\begin{array}{c}\text { joven } \\
\text { fonetista }\end{array}$ & $\begin{array}{c}\text { joven } \\
-\end{array}$ & $\begin{array}{c}\text { joven } \\
-\end{array}$ & $\begin{array}{c}\text { joven } \\
\text { fonetista }\end{array}$ & $\begin{array}{c}\text { mayor } \\
-\end{array}$ & $\begin{array}{c}\text { joven } \\
-\end{array}$ & $\begin{array}{c}\text { joven } \\
-\end{array}$ \\
\hline
\end{tabular}

Tabla 3.3. Comparación de las respuestas de los jueces de México por modalidad y por el origen del informante (A - Los Ángeles, B - México)

En el caso de los jueces que no son de México, añadir que las mayores dificultades se les han presentado en las aseverativas de B y en las interrogativas de A (marcado en rojo); justo lo contrario que les ha sucedido en las interrogativas de B y en las aseverativas de A (marcadas en verde), donde el nivel de errores es muy bajo. Resultados, por otro lado, totalmente lógicos, dado que la curva melódica de la frase aseverativa de Los Ángeles (con dos picos y una declinación moderada) y la curva melódica de la frase interrogativa de México (con perfil oblicuo) son las más generalizas en español. 


\begin{tabular}{|c|c|c|c|c|c|c|c|c|c|}
\hline \multicolumn{3}{|c|}{ NO MÉXICO } & NM1 & NM2 & NM3 & NM4 & NM5 & NM6 & NM7 \\
\hline \multirow{4}{*}{ 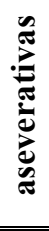 } & \multirow{2}{*}{$\mathbf{A x B}, \mathbf{x}=\mathbf{A}$} & Correctas & $94 \%$ & $100 \%$ & $100 \%$ & $94 \%$ & $100 \%$ & $94 \%$ & $89 \%$ \\
\hline & & Incorrectas & $6 \%$ & $0 \%$ & $0 \%$ & $6 \%$ & $0 \%$ & $6 \%$ & $11 \%$ \\
\hline & \multirow{2}{*}{$A x B, x=B$} & Correctas & $61 \%$ & $83 \%$ & $89 \%$ & $72 \%$ & $94 \%$ & $72 \%$ & $89 \%$ \\
\hline & & Incorrectas & $39 \%$ & $17 \%$ & $11 \%$ & $28 \%$ & $6 \%$ & $28 \%$ & $11 \%$ \\
\hline \multirow{4}{*}{ } & \multirow{2}{*}{$\mathrm{AxB}, \mathbf{x}=\mathbf{A}$} & Correctas & $83 \%$ & $83 \%$ & $72 \%$ & $89 \%$ & $94 \%$ & $72 \%$ & $100 \%$ \\
\hline & & Incorrectas & $17 \%$ & $17 \%$ & $28 \%$ & $11 \%$ & $6 \%$ & $28 \%$ & $0 \%$ \\
\hline & \multirow{2}{*}{$\mathbf{A x B}, \mathbf{x}=\mathbf{B}$} & Correctas & $89 \%$ & $100 \%$ & $89 \%$ & $100 \%$ & $89 \%$ & $89 \%$ & $83 \%$ \\
\hline & & Incorrectas & $11 \%$ & $0 \%$ & $11 \%$ & $0 \%$ & $11 \%$ & $11 \%$ & $17 \%$ \\
\hline
\end{tabular}

Tabla 3.4. Comparación de las respuestas de los jueces de No México por modalidad y por el origen del informante (A - Los Ángeles, B - México)

\section{CONCLUSIONES}

De los tres experimentos realizados, dos de ellos: test 1. identificación y test 3. discriminación $\mathrm{AXB}$, han demostrado un alto porcentaje de reconocimiento de los informantes de distintas zonas LA/Mx para ambas modalidades. Sin embargo, los resultados del test 2. discriminación simple revelan que este no ha sido tan eficaz, en consonancia con lo observado por otros investigadores en sus experimentos, por lo que requiere quizás un nuevo replanteamiento de su diseño para futuras pruebas.

También consideramos que sería necesario incorporar jueces mexicoamericanos para completar la validez de los resultados, así como aumentar el número global de jueces.

\section{REFERENCIAS BIBLIOGRÁFICAS}

Battaner, E., J. Gil, V. Marrero, J. Llisterri, C. Carbó, M. J. Machuca y A. RÍOS, (2003): «VILE: Estudio acústico de la variación inter e intralocutor en español», SEAF 2003, Actas del II Congreso de la Sociedad Española de Acústica Forense, Barcelona, Sociedad Española de Acústica, 59-70.

Boersma, Paul y David WeEnINK (2014): Praat: doing phonetics by computer [Computer program], versión 5.4 [en línea]: $<\underline{\mathrm{http}}: / /$ www.praat.org/>. [Consulta: 04/10/ 2014].

EsCUDERO, D. et al. (2003): «Uso de entonación en reconocimiento automático de locutor: Resultados preliminares», SEAF 2003, Actas del II Congreso de la Sociedad española de acústica forense, Barcelona, Sociedad Española de Acústica Forense, 167-174. 
Estebas Vilaplana, E. y P. PRieto Vives (2008): «La notación prosódica del español: una revisión del Sp_ToBI», Estudios de Fonética Experimental, vol. XVII, 263283.

Fernández Planas, Ana M.a (2005): «Datos generales del proyecto AMPER en España», Estudios de Fonética Experimental, vol. XIV, 13-27.

Fernández Planas, A. M. ${ }^{a}$, P. Roseano, W. Elvira-García, J. Carrera Sabaté, D. Roman Montes de OcA (en prensa): «From a Perceptual Point of View, Is There Prosodic Continuity between Languages in Contact?», en Congosto Martín, Y. y L. Morgenthaler, eds., Prosody and Language Contact in the Romance Languages.

INTERLANDI, G. y A. ROMANO (2004): «Le continuum intonatif de l'italien parlé à Turin: résultats d'un test d'identification», Actes du Workshop MIDL «Identification des langues et des varieties dialectales par les humains et par les machines», Presses de l'ENST, 157-60.

Liberman A. M., K. S. Harris, H. Hoffman y B. Griffith (1957): «The discrimination of speech sounds within and across phoneme boundaries», Journal of Experimental Psychology, vol. 54, 358-368, $<$ http://dx.doi.org/10.1037/h0044417>.

MARRERO, V. (2013): «Metodología de investigación en fonética perceptiva: posibles aportaciones para el estudio de la prosodia dialectal», Texnoloxías e análise dos datos lingüísticos. Simpos Metodoloxía io para o estudo perceptivo da variación prosódica dialectal [en línea]: <http://ilg.usc.es/tecandali/index.php/programa $>$. [Consulta: 10/01/2015].

Romano, A., M. Contini, J. P. LAi y A. Rilliard (2011): «Distancias prosódicas entre variedades románicas en el marco del proyecto AMPER», Revista Internacional de Lingüística Iberoamericana, vol. IX, núm. 1 (17), 13-25.

Simonet, M. (2012): «El diseño de experimentos para el estudio de la percepción del habla», taller impartido en Laboratory Approaches to Romance Phonology Conference, El Colegio de México, México D. F., 3 de octubre de 2012. [en línea]: $<$ http://sites.google.com/site/miquelsimonet/Home/scripts $>$. 